\title{
Physics of Open Fractures: Reconsidering Tissue Viability, Contamination Risk and Importance of Wound Debridement
}

\begin{abstract}
Ahmed Abdelmoez Alsayed
The Welsh Burn Centre for Burns \& Plastic Surgery, Morriston Hospital, Swansea Bay University Health Board, Swansea, UK Email: Ahmed.alsayed@wales.nhs.uk
\end{abstract}

How to cite this paper: Alsayed, A.A. (2021) Physics of Open Fractures: Reconsidering Tissue Viability, Contamination Risk and Importance of Wound Debridement. Journal of Applied Mathematics and Physics, 9, 176-182.

https://doi.org/10.4236/jamp.2021.91012

Received: December 6, 2020

Accepted: January 24, 2021

Published: January 27, 2021

Copyright $\odot 2021$ by author(s) and Scientific Research Publishing Inc. This work is licensed under the Creative Commons Attribution International License (CC BY 4.0).

http://creativecommons.org/licenses/by/4.0/

\begin{abstract}
Understanding basic physics behind open fractures leads to a better understanding of mechanism of injury, open fractures pathophysiology and management. Explaining local changes in viability of open fracture involved tissues, importance of debridement and reconsidering contamination risks will be ultimate objectives after going through this review. The controversy is still there between minimal/conservative debridement of open fracture wounds in favour of direct closure of soft tissue on the same session against generous debridement and delayed closure by more complicated choices on the reconstructive ladder to avoid infection, delayed healing, wound chronicity, limb loss and prolonged hospital stay. In the article, basic physics behind open fractures is highlighted to gain a deeper understanding of tissue viability changes and contamination risks after injury.
\end{abstract}

\section{Keywords}

Physics, Open Fractures, High Energy Trauma, Collision, Stress, Strain

\section{Introduction}

An open fracture is an injury where the fractured bone and/or fracture hematoma are exposed to the external environment through a traumatic defect of the soft tissue and skin. The skin wound may not lie directly over the fracture. Therefore, any fracture that has an associated wound should be considered open until proven otherwise [1].

Open fractures occur at a rate of 30.7 per 100,000 persons per year, most commonly due to motor vehicle accidents. The most common long bones fractured are tibia and fibula [1]. 


\section{Numerical Examples of High Energy Trauma}

If a car that weighs $1300 \mathrm{~kg}$ driven at a speed of 70 mile per hour (31.2928 meter per second) hits a standing still pedestrian. That car has a kinetic energy, just before hitting the victim, that can be calculated using the following equation:

$$
K=1 / 2 m v^{2}=1 / 2 \times 1300 \times(31.2928)^{2} \approx 636,505.5 \mathrm{~kg} \cdot \mathrm{m}^{2} / \mathrm{s}^{2}
$$

A bullet that weighs 100 grains (0.006479891 kilograms) shot from a close distance at a leg at a speed that equals 2600 feet per second (792.48 meter per second). The bullet kinetic energy will be:

$$
K=1 / 2 m v^{2}=1 / 2 \times 0.006479891 \times(792.48)^{2} \approx 2034.7 \mathrm{~kg} \cdot \mathrm{m}^{2} / \mathrm{s}^{2}
$$

A blow by a baseball bat that weighs 1 kilogram at a speed that equals 70 mile per hour (31.2928 meter per second) against a victim. The bat kinetic energy will be:

$$
K=1 / 2 m v^{2}=1 / 2 \times 1 \times(31.2928)^{2} \approx 489.6 \mathrm{~kg} \cdot \mathrm{m}^{2} / \mathrm{s}^{2}
$$

A suicidal victim that weighs 100 kilograms jumps of the roof of a five-storey building (15 metres). As gravitational acceleration approximately equals 9.81 $\mathrm{m} / \mathrm{s}^{2}$, his speed just before touching the ground will be $17.152 \mathrm{~m} / \mathrm{s}$. He will have a kinetic energy that equals the following just before touching the ground:

$$
K=1 / 2 m v^{2}=1 / 2 \times 100 \times(17.152)^{2} \approx 14,709.5 \mathrm{~kg} \cdot \mathrm{m}^{2} / \mathrm{s}^{2}
$$

$K$ is the kinetic energy, $m$ is the mass, $V$ is the velocity or speed. All the previously mentioned examples of high energy trauma show how different the intensity of a trauma can be, depending on their mechanisms and unique circumstances.

\section{Energy, Collision, Hooke’s Law}

In physics and chemistry, the law of conservation of energy states that the total energy of an isolated system remains constant and can neither be created nor destroyed; rather, it can only be transformed from a form to another, or transferred from an object to another within that system [2].

It is also important to note that mechanical energy of an object equals its kinetic and potential energies together [3].

When two objects collide and their total kinetic energy remains the same, it is called an elastic collision. In an ideal, perfectly elastic collision, there is no net conversion of kinetic energy into other forms such as heat, noise, or potential energy and there is no deformation of either objects involved in collision. That type of collision is more likely to happen on the atomic level.

In an inelastic collision, in contrast to an elastic collision, kinetic energy is not conserved as some of it turns into vibrational energy of the atoms, causing a heating effect, and the bodies are deformed. In collisions of macroscopic bodies, that rule applies.

Partially inelastic collisions are the most common form of collisions in the real world, like a car colliding with a pedestrian. In this type of collision, the objects 
involved in the collisions do not stick, but some kinetic energy is still lost. Friction, sound and heat are some ways the kinetic energy can be lost through partial inelastic collisions.

Based upon the previous three paragraphs, after a car collides with a pedestrian, it will:

1) Lose some of its kinetic energy, that will be transferred to the pedestrian mostly in the form of potential energy (Inelastic collision)

2) Keep some of its kinetic energy (continue moving even without pressing accelerator) and that means that inelastic collision was partial or imperfect [4].

Hypothetically speaking, if the collision exclusively affected the pedestrian lower leg, and as mentioned, it will lose some of its kinetic energy, that will be transferred to the pedestrian lower leg mostly in the form of potential energy (Inelastic collision), the collision will cause a stress against the integrity of lower leg tissues e.g. bone, muscle, skin, etc.

With oversimplification, stress value can be obtained by dividing the force applied by collision/the area of lower leg tissues affected by trauma.

$$
\sigma=F / A
$$

$\sigma$ is stress, $F$ is the force and $A$ is the surface area.

Lower leg tissues will deform momentarily. Strain the right term to describe the deformation happened.

If you try to stretch a slinky by grabbing both ends in opposite directions, you are applying a stress $(\sigma)$ on it. The change in the coil shape/length (deformation), while stretched, is the strain $(\varepsilon)$.

To a certain point of stretching called the yield strength point, the coil can show full elasticity and return back to its original shape when you let it go (area A under curve or elastic region).

If you stretch the slinky further to a certain point called ultimate strength point (area B under curve) and release the slinky, it will stay as one piece but will never return back to its original shape.

Further stretching (area $\mathrm{C}$ under curve) will cause necking of the slinky that will end in its fracture/breakage. If we add areas $\mathrm{A}, \mathrm{B}$ and $\mathrm{C}$ under the curve, the sum is known as the toughness (Figure 1).

Mathematically, the relation between stress and strain can be expressed using several terms according to the shape of the object, the surrounding environment and the nature and direction of forces applied on it (Figure 2). Young's modulus, shear modulus and several others are all products of generalized Hooke's law [6].

\section{Why Should We Debride All Open Fractures?}

\section{- Viability issues}

Four studies were chosen in this review to compare the stress/strain curve in the different tissues mainly involved in open fractures i.e. bone, skin, skeletal soft tissue and capillaries (Figure 3) [8] [9] [10] [11]. 


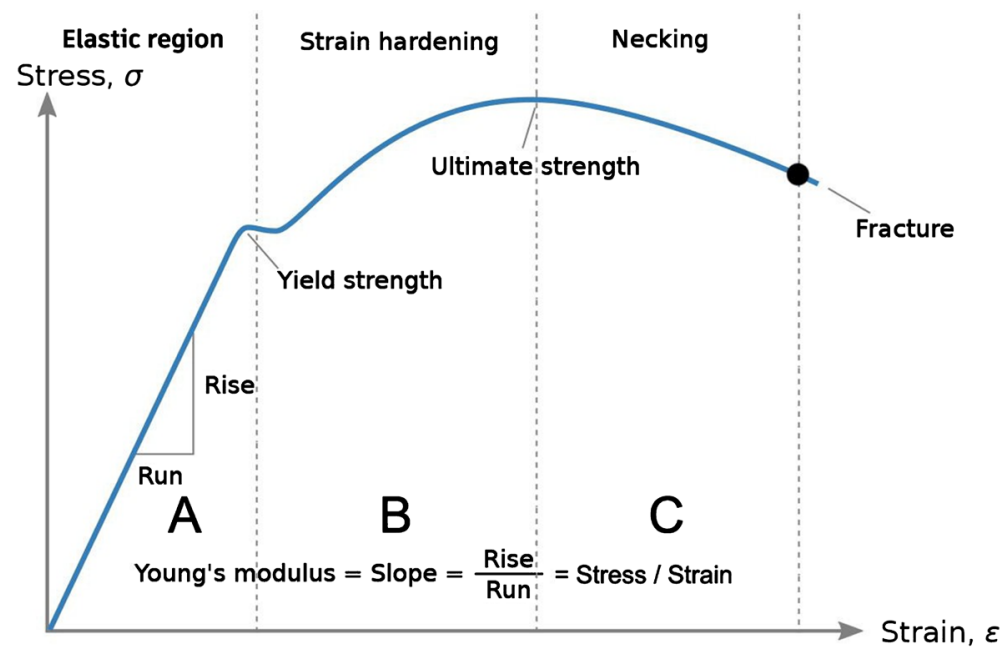

Figure 1. A typical stress-strain curve [5].

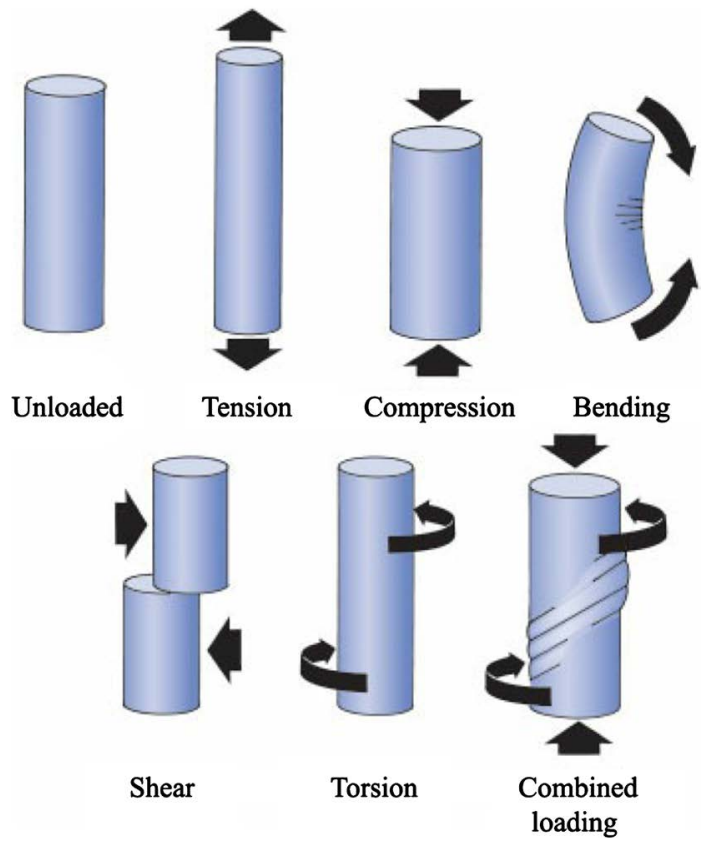

Figure 2. Schematic representation of various loading modes [7].

A trauma that can cause a fracture must be able to devastate the tissues surrounding bone such as skin, skeletal soft tissues and capillaries. That leads us to certain points:

1) Importance of universal tissue debridement in open fracture cases.

2) Gross skin integrity, around a puncture wound or a minor primary skin defect, at the open fracture site does not mean that the skin is viable. Skin may look intact. However capillary plexuses responsible for skin viability are completely damaged. In other words, skin becomes an apparently intact, though, dead tissue.

What just bothers bone, can devastate skin, skeletal soft tissues and capillaries.

Figure 3 compares stress/strain curves in four different tissues. However, if 

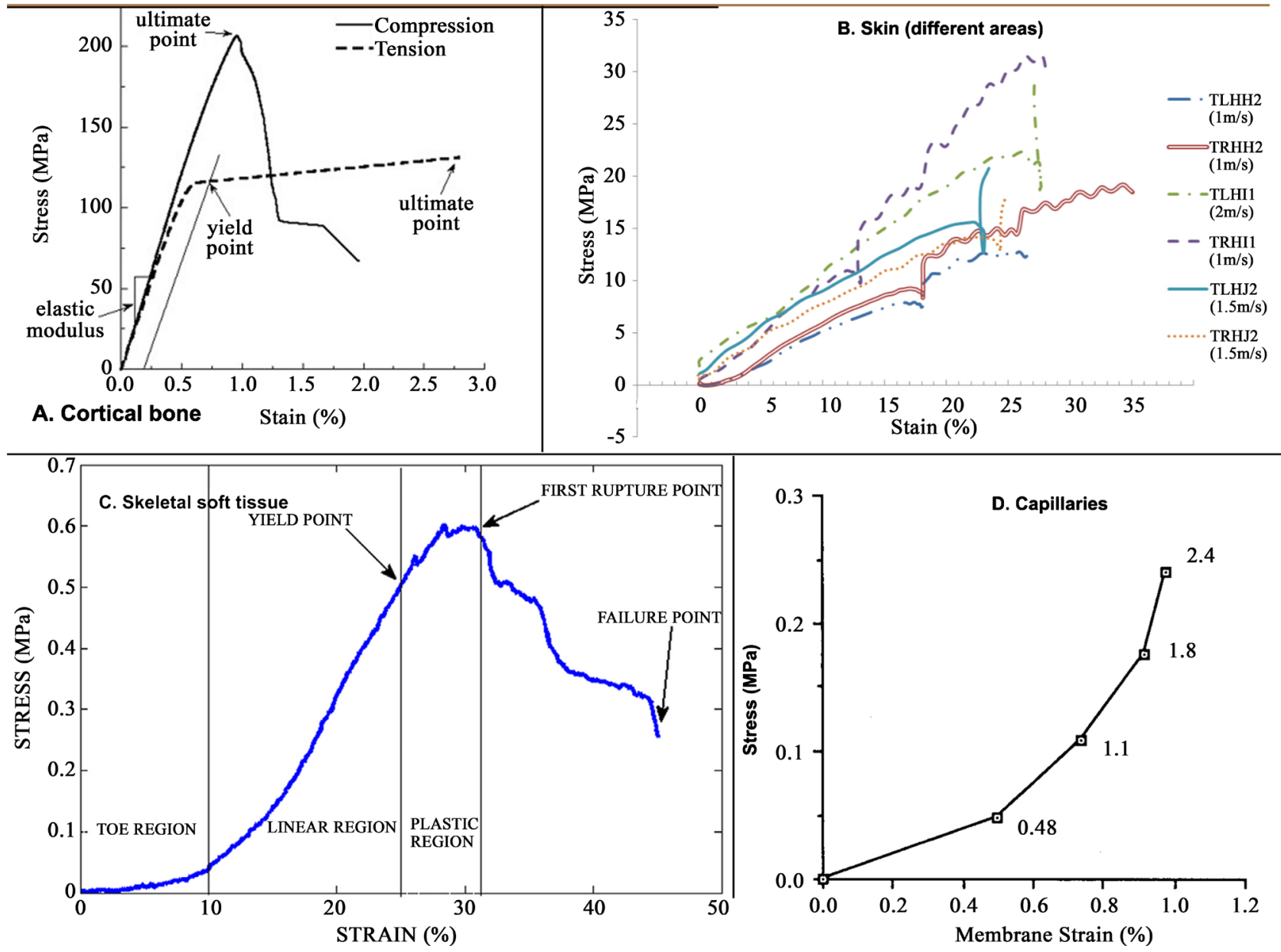

Figure 3. Comparison between stress/strain curves of (A) cortical bone, (B) skin, (C) skeletal soft tissues, (D) capillaries (supported by surrounding skeletal muscles). Measurement units are unified to show the different stresses required for different tissues break down/fracture [8] [9] [10] [11].

the same is tried to compare torque/angle deflection curves, that measures torsion modulus, results may change the order of tissues affected according to their different failure points. Moreover, physical properties differ in the same tissue type in different places in the same individual.

Trauma, in most cases, does not follow a single pattern per incidence. It is difficult to find an incidence where trauma was pure traction or pure torsion for all tissues for example. Most events comprise mixed patterns of trauma.

Based on the last two paragraphs, it is challenging in daily life examples of trauma to predict which tissues were damaged or survived. In a single example of a mixed traction torsion injury, it was found that bone, blood vessels and skin were damaged. However, muscles and tendons stayed grossly intact.

\section{- Contamination issues}

In cases of high energy trauma, energy is initially transferred into bone and soft tissues that targets tissues integrity through an expansile action on the tissues.

When you try to expand a compartment without adding a content inside it, 


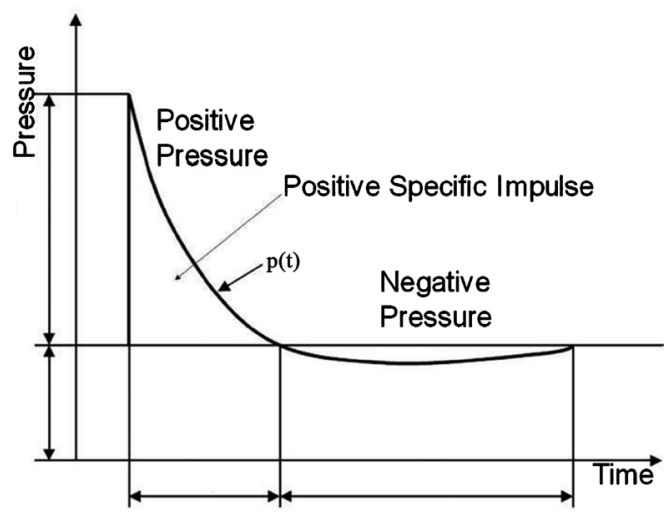

Figure 4. Typical Friedlander blast waveform [13].

pressure becomes negative inside that compartment making a vacuum effect [1] that encourages suction of external environmental components into the open fracture through the soft tissue defect causing contamination.

Friedlander was the first to describe that pattern or waveform in 1946, applying it mainly in blast injury cases (Figure 4). However, the mentioned pattern still applies in high energy trauma too. An open fracture with a tiny puncture wound doesn't exclude contamination chances [12].

\section{Conclusions}

The controversy is still there between minimal/conservative debridement of open fracture wounds in favour of direct closure of soft tissue on the same session against generous debridement and delayed closure by more complicated choices on the reconstructive ladder to avoid infection, delayed healing, wound chronicity, limb loss and prolonged hospital stay.

Understanding physics behind open fracture leads to a better understanding of mechanism of injury, open fractures pathophysiology and management. Moreover, it gives a bigger picture of tissue viability changes and contamination risks after injury.

Wound excision/debridement in all open fracture cases and staged management, if required, is necessary to confirm that tissues are viable and contamination free.

\section{Conflicts of Interest}

The author declares no conflicts of interest regarding the publication of this paper.

\section{References}

[1] Sop, J.L. and Sop, A. (2020) Open Fracture Management. StatPearls Publishing, Treasure Island (FL). https://www.ncbi.nlm.nih.gov/books/NBK448083/

[2] Feynman, R. (1970) The Feynman Lectures on Physics Vol I. Addison Wesley.

[3] Newton, I., Cohen, I.B. and Whitman, A.M. (Eds.) (1999) The Principia: Mathematical Principles of Natural Philosophy. University of California Press, Berkeley. 
[4] Tolman, R.C. (1938) The Principles of Statistical Mechanics. Clarendon Press, Oxford. (Reissued, 1979, Dover, New York)

[5] Nicoguaro, Typical Stress vs. Strain Diagram for a Ductile Material (e.g. Steel). Wikimedia Commons, the Free Media Repository.

[6] Hooke, R. (1678) De Potentia Restitutiva, or of Spring. Explaining the Power of Springing Bodies, London.

[7] Nordin, M. and Frankel, V.H. (2012) Basic Biomechanics of the Musculoskeletal System. 4th Edition, Chapter 2: Biomechanics of Bone. Lippincott Williams \& Wilkins, a Wolters Kluwer Business, Baltimore, 84-85.

[8] Morgan, E.F., Lee, J.J. and Keaveny, T.M. (2005) Sensitivity of Multiple Damage Parameters to Compressive Overload in Cortical Bone. Journal of Biomechanical Engineering, 127, 557-562. https://doi.org/10.1115/1.1933916

[9] Gallagher, A.J., Ní Annaidh, A. and Bruyère-Garnier, K. (2012) Dynamic Tensile Properties of Human Skin. 2012 IRCOBI Conference Proceedings, 12-14.

[10] Gaur, P., Chawla, A., Verma, K., Mukherjee, S., Lalvani, S., Malhotra, R., et al. (2016) Characterisation of Human Diaphragm at High Strain Rate Loading. Journal of the Mechanical Behavior of Biomedical Materials, 60, 603-616. https://doi.org/10.1016/j.jmbbm.2016.02.031

[11] Lee, J. and Schmid-Schönbein, G.W. (1995) Biomechanics of Skeletal Muscle Capillaries: Hemodynamic Resistance, Endothelial Distensibility, and Pseudopod Formation. Annals of Biomedical Engineering, 23, 226-246. https://doi.org/10.1007/BF02584425

[12] Friedlander, F. (1946) The Diffraction of Sound Pulses; Diffraction by a Semi-Infinite Plane. Proceedings of the Royal Society of London. Series A, Mathematical and Physical Sciences, 186, 322-344. https://doi.org/10.1098/rspa.1946.0046

[13] Raj, K. and Andrzej, E. (2013) Mathematical Models of Blast Induced TBI: Current Status, Challenges and Prospects. Frontiers in Neurology, 4.

https://doi.org/10.3389/fneur.2013.00059 